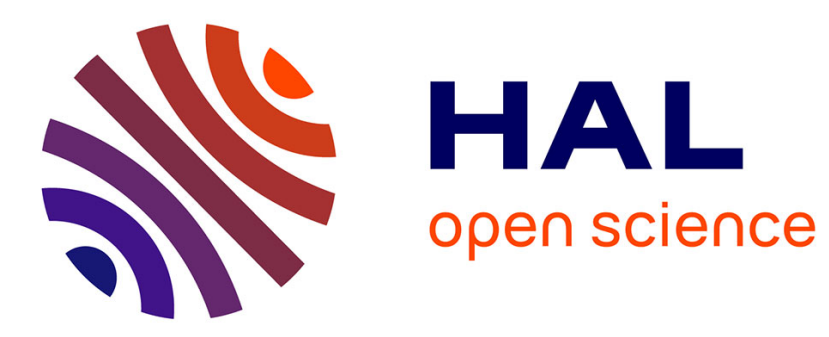

\title{
Robust Domain of Attraction Estimation for a Tumor Growth Model
}

\author{
Kaouther Moussa, Mirko Fiacchini, Mazen Alamir
}

\section{To cite this version:}

Kaouther Moussa, Mirko Fiacchini, Mazen Alamir. Robust Domain of Attraction Estimation for a Tumor Growth Model. Applied Mathematics and Computation, 2021, 410, pp.126482. 10.1016/j.amc.2021.126482 . hal-03359186

\section{HAL Id: hal-03359186 \\ https://hal.science/hal-03359186}

Submitted on 30 Sep 2021

HAL is a multi-disciplinary open access archive for the deposit and dissemination of scientific research documents, whether they are published or not. The documents may come from teaching and research institutions in France or abroad, or from public or private research centers.
L'archive ouverte pluridisciplinaire $\mathbf{H A L}$, est destinée au dépôt et à la diffusion de documents scientifiques de niveau recherche, publiés ou non, émanant des établissements d'enseignement et de recherche français ou étrangers, des laboratoires publics ou privés. 


\title{
Robust Domain of Attraction Estimation for a Tumor Growth Model
}

\author{
Kaouther Moussa*, Mirko Fiacchini* and Mazen Alamir*
}

November 3, 2020

\begin{abstract}
This paper deals with the estimation of regions of attraction (RoAs) for a cancer dynamical model. The estimation of this type of sets is very interesting in the field of control for cancer dynamics, since it provides the set of possible initial health indicators, for which there exists a treatment protocol allowing to heal the patient. Therefore, we propose a methodology to estimate the region of attraction of a nonlinear dynamical system describing the interaction between a tumor, the immune system and combined therapies of cancer. Then, we use the characterization of the RoA for a given model parameters vector, in order to derive an estimation of an outer approximation of the robust RoA under parametric uncertainties.
\end{abstract}

Estimating the region of attraction (RoA) of equilibrium points is a fundamental problem in systems engineering [6]. This set, called also the domain or the basin of attraction, contains the initial states that can be driven to a stable equilibrium point, without violating the specified constraints. Therefore, the estimation of regions of attraction is a very important and still open field of research [2].

In practical problems, the systems are often affected by different types of uncertainties. Hence, one of the challenging problems in the control of dynamical systems is the estimation of robust regions of attraction for nonlinear and uncertain systems. According to [4], the Lyapunov theory for ODEs initiated the notion of invariant sets for control problems. Deriving the exact RoA for dynamical systems is a challenging task, therefore, researchers focus on determining Lyapunov functions, since the sublevel sets of the latter represent the boundaries of positively invariant sets [29]. In fact, a positively invariant set, for a given dynamical system, is such that if it contains the states at a given time, then, there is a guarantee that it will contain the state trajectories for the future.

One of the commonly used convex sets for the estimation of invariant sets are polyhedrons and ellipsoids. According to [2], invariant ellipsoids have been used in the literature in order to estimate the regions of attraction of nonlinear systems. In [4], a detailed review

\footnotetext{
${ }^{*}$ Univ. Grenoble Alpes, CNRS, Grenoble INP, GIPSA-lab, 38000 Grenoble, France \{kaouther.moussa, mirko.fiacchini, mazen.alamir\}@grenoble-inp.fr
} 
on invariant sets approaches is provided, with a specific comparison between polyhedrons and ellipsoids, in terms of estimation accuracy and flexibility. According to [5], it is established, in terms of RoA estimation as well as robustness analysis, that the ellipsoidal based approaches are conservative. In contrast to ellipsoids, polyhedral sets provide less conservative solutions, although they might be computationally expensive.

The estimation of regions of attraction for linear systems has received a specific attention in the literature. There exist many works for this class of systems, see for example [3], [4], [5] and [31]. In contrast to linear systems, the characterization of regions of attraction for nonlinear systems is an open research topic. There exist some approaches, that are based on convex difference inclusions (CDIs), allowing to estimate the RoAs for nonlinear systems, see [1], [11], [10], [13], [12]. Furthermore, in [24], the latter methods were extended to characterize the RoAs for nonlinear systems, subject to different types of uncertainties.

Moreover, there are other methods based on the moment optimization framework, allowing to estimate the RoAs of polynomial dynamical systems and providing a hierarchy of semi-algebraic outer (or inner) approximations of the ROA, by solving a sequence of linear matrix inequalities (LMIs) problems, see [16] and [15]. However, as mentioned in [21] and [22], the moment optimization based methods are limited to low dimensional systems and require a relatively high computational time. Therefore, extending these approaches to uncertain systems might be challenging.

In the context of cancer treatment, the regions of attraction are interpreted as the sets of initial health conditions (tumor volume and immune cells density for example), for which there exists a treatment strategy such that the patient recovers, without any health damage or side effect. Therefore, the characterization of this type of sets is essential for the analysis of cancer related dynamical systems. Furthermore, since this class of systems is known to be highly uncertain, it is crucial to estimate the RoA under uncertainties for such systems.

There exist in the literature few works regarding the estimation of RoAs and robust RoAs for cancer dynamical systems. We cite for example [7] and [30], where the authors proposed different Lyapunov functions based approaches, to estimate the domain of attraction of the tumor free equilibrium point corresponding to autonomous cancer growth models, where no therapies are considered, see also [19] and references therein. Furthermore, in [25], an iterative procedure method, based on approximating the uncertain system with CDIs, was presented to estimate the robust region of attraction of a tumor growth model with chemotherapy. However, the model that we consider in this paper has not been investigated in the literature to estimate its controlled region of attraction.

In this paper, we propose a readily applicable methodology that is in the same line of sliding mode control, in order to characterize the region of attraction of a cancer dynamical model, using bang-bang control strategies. Furthermore, this methodology will 
be used in order to derive an estimate of the robust region of attraction, where the model parameters are considered to be uncertain. It is worth emphasizing that this approach does not provide the control strategies to be applied, however, it provides the set of initial conditions, such that for every initial condition in this set, there exists a control strategy allowing to drive the states to a benign stable equilibrium. This can also be seen as to provide an estimate of the control invariant set corresponding to the benign stable equilibrium.

This paper is organized as follows: In Section 1, we present the cancer dynamical model, furthermore, we investigate the parametric space of this model and we analyze the effects of parametric uncertainties on the model equilibrium points. In Section 2, we present the methodology allowing to derive the RoA of the cancer benign equilibrium, corresponding to the considered model. We use the latter approach in Section 3 in order to derive an estimation of the robust RoA. Finally, Section 4 summarizes the work that we present in this paper.

\section{Dynamical model}

We consider here the same model as in [22], describing the interaction between a tumor and the immune system under the effects of a combined therapy:

$$
\begin{aligned}
\dot{x}_{1} & =\mu_{C} x_{1}-\frac{\mu_{C}}{x_{\infty}} x_{1}^{2}-\gamma_{X} x_{1} x_{2}-\kappa_{X} x_{1} u_{1}, \\
\dot{x}_{2} & =\mu_{I} x_{1} x_{2}-\beta_{Y} x_{1}^{2} x_{2}-\delta_{Y} x_{2}+\kappa_{Y} x_{2} u_{2}-\eta_{Y} u_{1} x_{2}+\alpha_{Y}, \\
x(0) & =\left(x_{1}(0), x_{2}(0)\right)=x_{0},
\end{aligned}
$$

where $x_{1}$ and $x_{2}$ denote, respectively, the number of tumor cells and the density of effector immune cells (ECs), $u_{1}$ and $u_{2}$ are, respectively, the delivery profiles of a cytotoxic agent (chemotherapy) and an immunostimulator. The initial state of system (1) is denoted by $x_{0}$.

The model that we investigate here is a modified version of the Stepanova model [28] that has been extensively used in the literature, we cite for example [8], [17] and [18] where optimal control approaches were proposed to schedule chemo- and immunotherapy injection profiles. Furthermore, [26] proposed a multiple model predictive control scheme to design chemo- and immunotherapy injection schedules. Moreover, in [27], the authors proposed a robust multiple model predictive control scheme for this model, in order to consider direct drug targeting pharmacokinetic uncertainties as well as system model mismatches. Although this model has been widely used, it has never been investigated in the literature to estimate the controlled region of attraction of its corresponding tumor free equilibrium.

Table 1 summarizes the definitions of the model parameters and their nominal values. We slightly changed the values of some parameters since with the previous set of parameters values (used in [21] and [22] and taken from [8]), the domain of attraction for the 
uncontrolled system (1) (for $u_{1}=0$ and $u_{2}=0$ ) was unrealistically big. This allows us to solve a problem which is more reasonable and realistic from a practical point of view. Furthermore, we focus on the assessment of a methodology that remains applicable for different nominal parameters values.

Table 1: Definitions and nominal values of the parameters used in model (1).

\begin{tabular}{|c|c|c|}
\hline Parameter & Definition & Numerical value \\
\hline$\mu_{C}$ & tumor growth rate & $1.0078 \cdot 10^{7}$ cells $/$ day \\
\hline$\mu_{I}$ & $\begin{array}{l}\text { tumor stimulated } \\
\text { proliferation rate }\end{array}$ & $0.0029 \mathrm{day}^{-1}$ \\
\hline$\alpha_{Y}$ & $\begin{array}{l}\text { rate of immune } \\
\text { cells influx }\end{array}$ & 0.0827 day $^{-1}$ \\
\hline$\beta_{Y}$ & inverse threshold & 0.0040 \\
\hline$\gamma_{X}$ & interaction rate & $1 \cdot 10^{7}$ cells $/$ day \\
\hline$\delta_{Y}$ & death rate & 0.1873 day $^{-1}$ \\
\hline$\kappa_{X}$ & chemotherapeutic & $1 \cdot 10^{7}$ cells/day \\
\hline$\kappa_{Y}$ & $\begin{array}{l}\text { killing parameter } \\
\text { immunotherapy } \\
\text { injection parameter }\end{array}$ & $1 \cdot 10^{7}$ cells /day \\
\hline$x_{\infty}$ & fixed carrying capacity & $780 \cdot 10^{6}$ cells \\
\hline$\eta_{Y}$ & $\begin{array}{l}\text { chemo-induced loss } \\
\text { on immune cells }\end{array}$ & 1 \\
\hline
\end{tabular}

Let's denote by $x=\left(x_{1}, x_{2}\right)$ and $u=\left(u_{1}, u_{2}\right)$, respectively, the state and the control input vectors. The uncontrolled nominal model (1) (for $u=(0,0)$ ) has two locally asymptotically stable equilibrium points. The macroscopic malignant equilibrium is $x_{m}=(766.44,0.08)$ and the benign one is $x_{b}=(41.45,0.95)$.

In standard control problems for cancer dynamics, the objective of the treatment consists in general in driving the state trajectories from the region of attraction of the malignant equilibrium to the region of attraction of the benign equilibrium. This can be seen as to switch an acute tumor to its chronic state. In this paper, we are interested in characterizing the set of initial conditions (tumor volume and immune density) from which the state trajectories can be driven to the safe region.

In the context of cancer treatment, the determination of the region of attraction is an interesting problem, since it provides an information on the possibility of recovery for a patient, given the initial measured health conditions. We mean by recovery reaching a safe region where the tumor is considered to be harmless, and there is no need to inject drugs. The safe region corresponds to the region of attraction of the locally asymptotically stable benign equilibrium $x_{b}$ without therapies. This set as well as the region of 
attraction under treatment will be properly defined in the sequel.

Moreover, we will use the characterization of the domain of attraction of system (1) to derive an estimate of the robust region of attraction when the model parameters are considered to be uncertain and belong to a given hyperbox.

In this section, we will provide necessary and sufficient conditions for the equilibriums of system (1) to exist, given the vector of model parameters $p$. We will also investigate the parametric space and show the equilibrium points distributions. Furthermore, we will provide an estimate of the region of attraction of the benign equilibrium $x_{b}$ when nominal parameters are considered (the parameters values in Table 1).

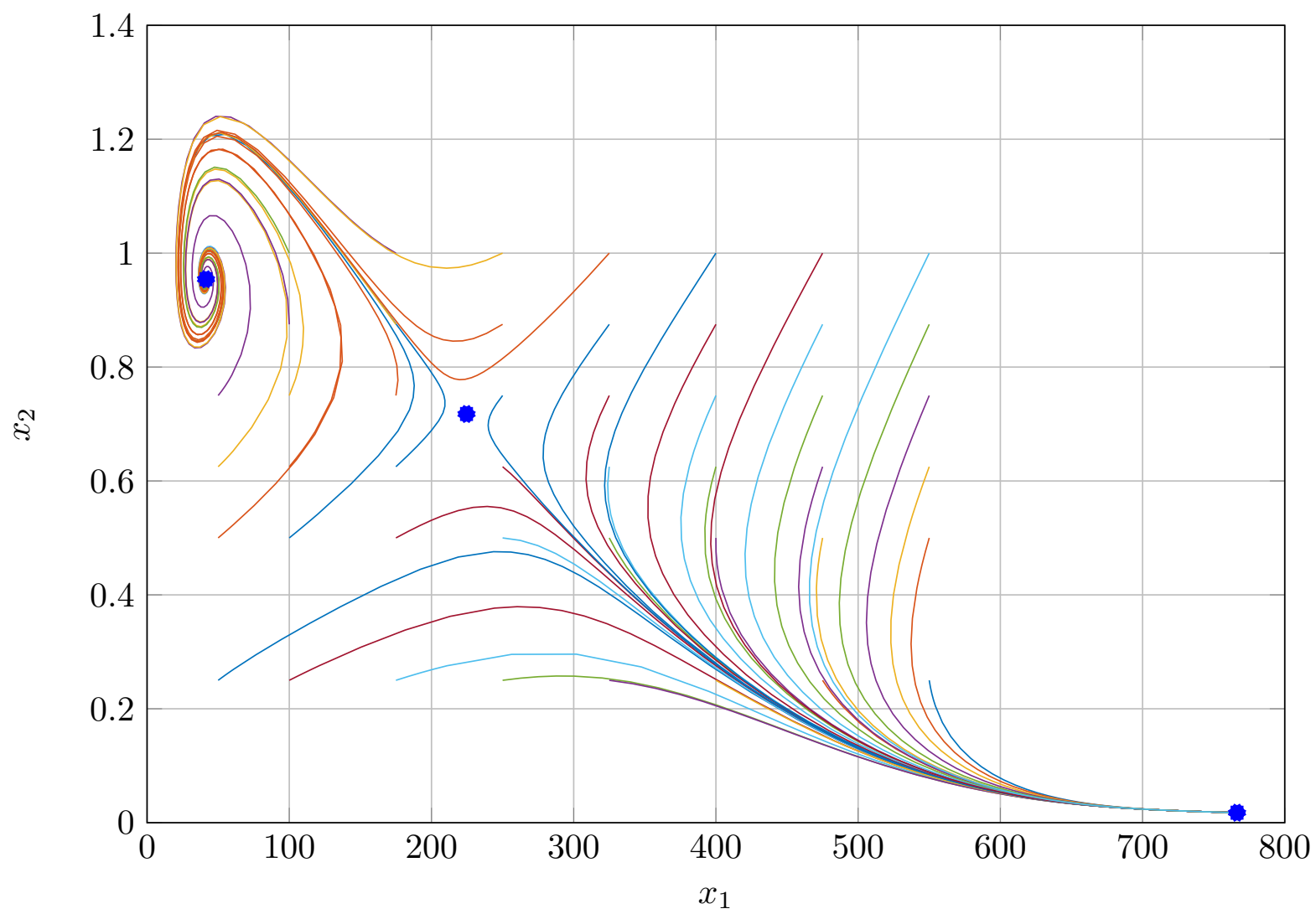

Figure 1: Phase portrait of (1) with the three equilibrium points.

\subsection{Model equilibriums}

We are interested in finding a general equation to obtain the equilibrium points of model (1) when no control is applied $(i e: u=(0,0))$. Therefore, we need to solve the following equations:

$$
\begin{aligned}
& \dot{x}_{1}=\mu_{C} x_{1}-\frac{\mu_{C}}{x_{\infty}} x_{1}^{2}-\gamma_{X} x_{1} x_{2}=0, \\
& \dot{x}_{2}=\mu_{I}\left(x_{1}-\beta_{Y} x_{1}^{2}\right) x_{2}-\delta_{Y} x_{2}+\alpha_{Y}=0 .
\end{aligned}
$$


The nontrivial solution of $(2)$ is:

$$
x_{1}=\frac{x_{\infty}}{\mu_{C}}\left(\mu_{C}-\gamma_{X} x_{2}\right) .
$$

By replacing (4) in (3), we obtain that solving $\dot{x}_{2}=0$ implies solving the following polynomial equation:

$$
-\frac{\mu_{I} \beta_{Y} x_{\infty}^{2} \gamma_{X}^{2}}{\mu_{C}^{2}} x_{2}^{3}+\left(\frac{2 \mu_{I} \beta_{Y} x_{\infty}^{2} \gamma_{X}-\mu_{I} x_{\infty} \gamma_{X}}{\mu_{C}}\right) x_{2}^{2}+\left(\mu_{I} x_{\infty}-\mu_{I} \beta_{Y} x_{\infty}^{2}-\delta_{Y}\right) x_{2}+\alpha_{Y}=0 .
$$

We denote by $a\left(x_{2}\right)$ the monic polynomial corresponding to the polynomial in (5) as follows:

$a\left(x_{2}\right)=x_{2}^{3}+\mu_{C}\left(\frac{\mu_{I} x_{\infty} \gamma_{X}-2 \mu_{I} \beta_{Y} x_{\infty}^{2} \gamma_{X}}{\mu_{I} \beta_{X} x_{\infty}^{2} \gamma_{X}^{2}}\right) x_{2}^{2}+\mu_{C}^{2}\left(\frac{\delta_{Y}+\mu_{I} \beta_{Y} x_{\infty}^{2}-\mu_{I} x_{\infty}}{\mu_{I} \beta_{Y} x_{\infty}^{2} \gamma_{X}^{2}}\right) x_{2}-\frac{\mu_{C}^{2} \alpha_{Y}}{\mu_{I} \beta_{Y} x_{\infty}^{2} \gamma_{X}^{2}}$.

This notation will be used in the sequel in order to investigate the parametric space corresponding to model (1).

Considering the nominal parameters in Table 1, the polynomial (5) has three real solutions. The state $x_{1}$ corresponding to the number of tumor cells can be obtained through (4) for each root of (5). The three equilibriums of system (1) are the benign and the malignant ones, which are locally asymptotically stable, and the saddle point which separates the regions of attraction of the benign and malignant equilibriums (see Figure 1).

\subsection{Estimating the domain of attraction of the benign equilib- rium}

Let's denote by $p \in \mathbb{P} \subset \mathbb{R}_{+}^{n_{p}}$ the vector of dimension $n_{p}=9$ containing the parameters of model (1) such that:

$$
p=\left(\mu_{C}, \mu_{I}, \alpha_{Y}, \beta_{Y}, \gamma_{X}, \delta_{Y}, \kappa_{X}, \kappa_{Y}, \eta_{Y}\right)^{T}
$$

The uncontrolled system (1) can be written in the following form:

$$
\dot{x}=F(x, p), \quad x(0)=x_{0},
$$

where $x_{0}$ stands for the initial state.

Let $\phi\left(t, x_{0}, p\right)$ be the solution of (8) evaluated at time $t \geq 0$ and corresponding to the state initial condition $x_{0}$ and the parameters vector $p$. We denote by $x_{b}^{p}$ the benign equilibrium of system (1) for a given parameters vector $p$. Note that the existence of a benign equilibrium depends on the vector of parameters $p$. We will provide in the sequel necessary and sufficient conditions for the existence of such an equilibrium. 
Definition 1 The RoA $\Omega_{0}^{p}$ of the benign equilibrium of the uncontrolled system (8) for a given parameters vector $p$ is defined as follows:

$$
\Omega_{0}^{p}=\left\{x_{0} \in \mathbb{R}_{+}^{2} \mid \lim _{t \rightarrow \infty} \phi\left(t, x_{0}, p\right)=x_{b}^{p}\right\} .
$$

The region of attraction $\Omega_{0}^{p}$ characterizes the set of initial states that can be driven to the benign equilibrium without any control action. This set can be seen as the safe region previously explained, since there is a guarantee that all trajectories having as initial state $x_{0} \in \Omega_{0}^{p}$, converge to the benign equilibrium $x_{b}^{p}$ after some time, and without control. Therefore, $\Omega_{0}^{p}$ can be used as a target set for any control strategy.

Let's denote by $p_{\text {nom }} \in \mathbb{R}_{+}^{n_{p}}$ the vector containing the nominal parameters of model (1) (presented in Table 1), such that:

$$
p_{\text {nom }}=(1.0078,0.0029,0.0827,0.004,1,0.1873,1,1,1)^{T} .
$$

As mentioned in [8], finding an analytic description for the domain of attraction of the benign equilibrium denoted $\Omega_{0}^{p_{\text {nom }}}$ might be challenging. However, there exist some methods for approximating these sets, see for example [14] and [9].

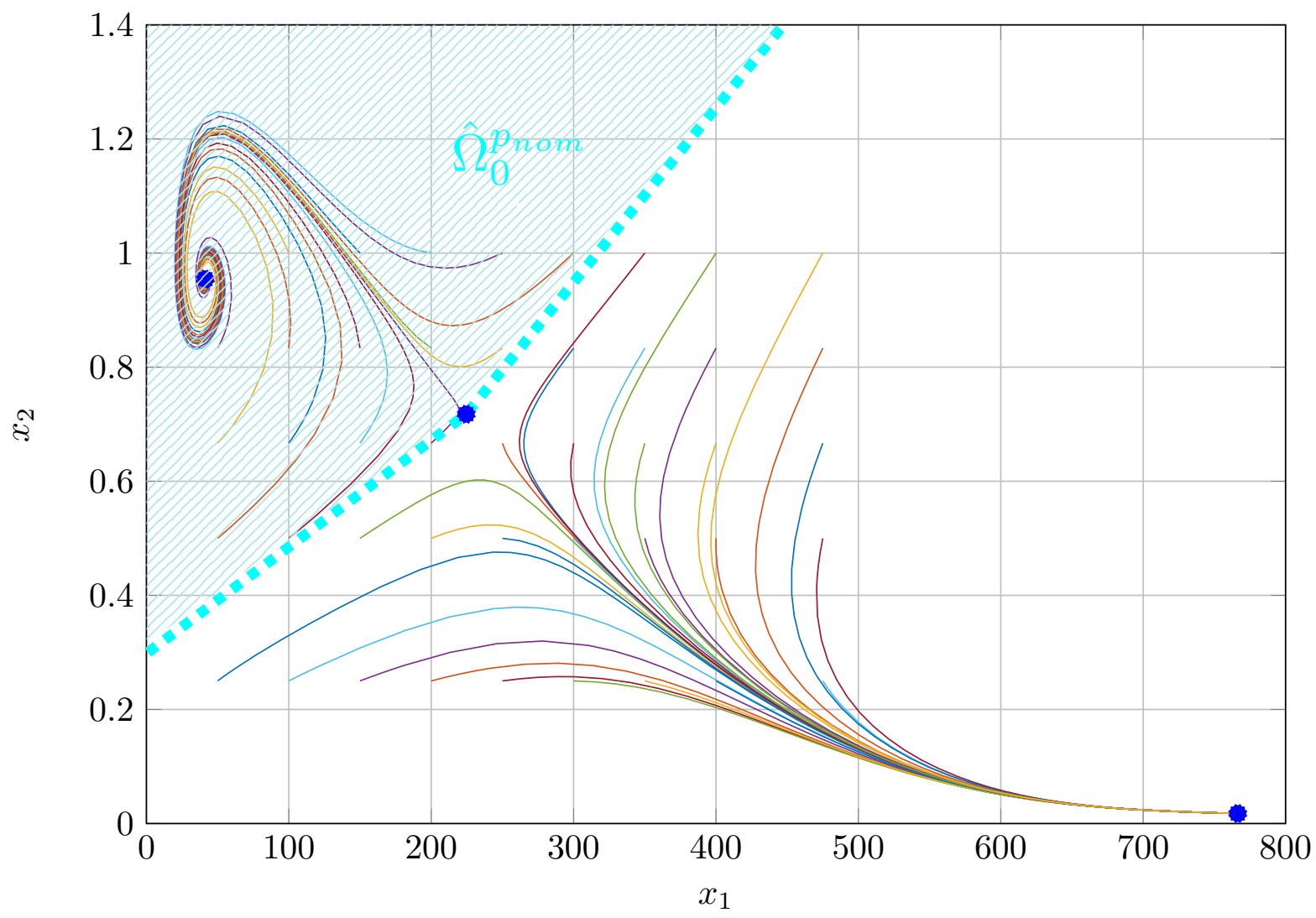

Figure 2: Phase portrait of (1) with nominal parameters $p_{\text {nom }}$, estimate of the nominal uncontrolled RoA of the benign equilibrium $\hat{\Omega}_{0}^{p_{\text {nom }}}$ in dashed cyan.

Definition 2 We denote by $\hat{\Omega}_{0}^{p_{\text {nom }}}$ an estimate of the nominal uncontrolled RoA of the benign equilibrium denoted $\Omega_{0}^{p_{\text {nom }}}$. 
Note that $x_{b}^{p_{\text {nom }}}$ is the same previously defined benign equilibrium point $x_{b}=(41.45,0.95)$, when nominal parameters are considered.

Figure 2 shows the phase portrait of system (1) with an estimation of the nominal uncontrolled region of attraction of the benign equilibrium. This set is considerably smaller than the region of attraction of the benign equilibrium with the previous set of parameters used in [22].

In the context of standard control, where deterministic parameters are considered, the set shown in Figure 2 can be used as a target set for the defined control strategy, since all the trajectories starting in this set converge to the corresponding benign equilibrium without any control action.

\subsection{Parametric space investigation}

In the previous section, we presented the general equations providing the equilibriums of system (1). The roots of the polynomial (6) can be either real or complex depending on the parameters vector $p$. In this paper, we are interested in providing an estimation of the robust region of attraction of system (1) subject to parametric uncertainties. Therefore, it is interesting to investigate the parametric space, since there might be some inadmissible parameter vectors, for which the polynomial (6) has complex roots, this case being unrealistic in the context of cancer dynamics modeling.

In the sequel, we provide necessary and sufficient conditions for system (1) to have real distinct equilibrium points. Furthermore, we illustrate these conditions with examples in both cases, when the polynomial equation allowing to derive the equilibrium points of system (1) has only real roots, as well as in the case when it has complex roots.

Theorem 1 The system (1) for a given parameters vector $p$ has three real distinct equilibrium points if and only if the following condition is satisfied:

$$
\mathcal{H}\left(a_{p}\right):=\left(\begin{array}{lll}
s_{0} & s_{1} & s_{2} \\
s_{1} & s_{2} & s_{3} \\
s_{2} & s_{3} & s_{4}
\end{array}\right) \succ 0
$$

where $a_{p}$ stands for the coefficients vector corresponding to the polynomial $a\left(x_{2}\right)(6)$, and $\mathcal{H}\left(a_{p}\right)$ denotes the Hermit matrix of the polynomial $a\left(x_{2}\right)$. The coefficients of the 
Hermit matrix $s_{0}, s_{1}, s_{2}, s_{3}$ and $s_{4}$ have the following expressions:

$$
\left\{\begin{array}{l}
s_{0}=3 \\
s_{1}=\mu_{C}\left(\frac{2 \beta x_{\infty}-1}{\beta_{Y} x_{\infty} \gamma_{X}}\right) \\
s_{2}=\frac{\mu_{C}^{2}}{\mu_{I} \beta_{Y}^{2} x_{\infty}^{2} \gamma_{X}^{2}}\left(\mu_{I}-2 \delta_{Y} \beta_{Y}-2 \mu_{I} \beta_{Y} x_{\infty}+2 \mu_{I} \beta_{Y}^{2} x_{\infty}^{2}\right) \\
s_{3}=\frac{\mu_{C}^{2}}{\mu_{I} \beta_{Y}^{3} x_{\infty}^{3} \gamma_{X}^{3}}\left(-\mu_{C} \mu_{I}+3 \mu_{C} \mu_{I} \beta_{Y} x_{\infty}-3 \mu_{C} \mu_{I} \beta_{Y}^{2} x_{\infty}^{2}+8 \mu_{C} \mu_{I} \beta_{Y}^{3} x_{\infty}^{3}+3 \mu_{C} \beta_{Y} \delta_{Y}\right. \\
\left.\quad-6 \mu_{C} \mu_{I} \beta_{Y}^{2} x_{\infty} \delta_{Y}-6 \mu_{C} \mu_{I}^{2} \beta_{Y}^{3} x_{\infty}^{3}+3 \alpha_{Y} \beta_{Y}^{2} x_{\infty} \gamma_{X}\right) \\
s_{4}=\left(1-4 \beta_{Y} x_{\infty}+4 \beta_{Y}^{2} x_{\infty}\right)\left(\frac{\mu_{C}^{4}\left(1-4 \beta_{Y} x_{\infty}+4 \beta_{Y}^{2} x_{\infty}^{2}\right)}{\beta_{Y}^{4} x_{\infty}^{4} \gamma_{Y}^{4}}-\frac{4 \mu_{C}^{4}\left(\delta_{Y}+\mu_{I} \beta_{Y} x_{\infty}^{2}-\mu_{I} x_{\infty}\right)}{\mu_{I} \beta_{Y}^{3} x_{\infty} x_{\infty}^{4} \gamma_{X}^{4}}\right)
\end{array}\right.
$$

The proof of Theorem 1 is given in Appendix A. The condition (11) provided by Theorem 1 is satisfied if and only if the eigenvalues of the Hermit matrix $\mathcal{H}\left(a_{p}\right)$ are strictly positive. Let's denote by $\Lambda$ the vector containing the eigenvalues of the Hermit matrix $\mathcal{H}\left(a_{p}\right)$.

Example 1 Considering the vector of nominal parameters $p_{\text {nom }}$ defined in Table (1), we check the condition in Theorem 1 by computing the eigenvalues of the corresponding Hermit matrix:

$$
\Lambda=\left(\begin{array}{l}
0.9543 \\
0.7176 \\
0.0175
\end{array}\right)
$$

we can notice that the condition of Theorem 1 is satisfied, which is directly related to the fact that system (1) has the three real distinct equilibrium points $(41.45,0.95),(224.64,0.72)$ and (766.44, 0.02) (see Figure 1).

Example 2 Let's consider the following parameters:

$$
\left\{\begin{array}{l}
\mu_{C}=1.1497 \\
\mu_{I}=0.0024 \\
\delta_{Y}=0.2210 \\
\alpha_{Y}=0.0739 \\
\beta_{Y}=0.0046 \\
\gamma_{X}=1.0391 \\
x_{\infty}=780
\end{array}\right.
$$

In this case, we obtain that the Hermit matrix has the following eigenvalues:

$$
\Lambda=\left(\begin{array}{c}
-0.003 \\
0.5902 \\
6.1589
\end{array}\right)
$$


which does not satisfy the condition in Theorem 1 since we have one negative eigenvalue. The roots of polynomial (6) obtained for this set of parameters are the following:

$$
x_{2}=\left(\begin{array}{l}
0.98 \pm 0.06 i \\
0.02
\end{array}\right)
$$

In this case, we have only one real equilibrium point $(769.80,0.02)$ corresponding to the malignant equilibrium. Figure 3 shows the phase portrait corresponding to the set of parameters considered in this example. We can see that this phase portrait does not have the same characteristics as in Figure 1, where we have two locally asymptotically stable equilibria, the benign one corresponding to an acute tumor and the malignant one corresponding to its chronic state as well as the real saddle point.

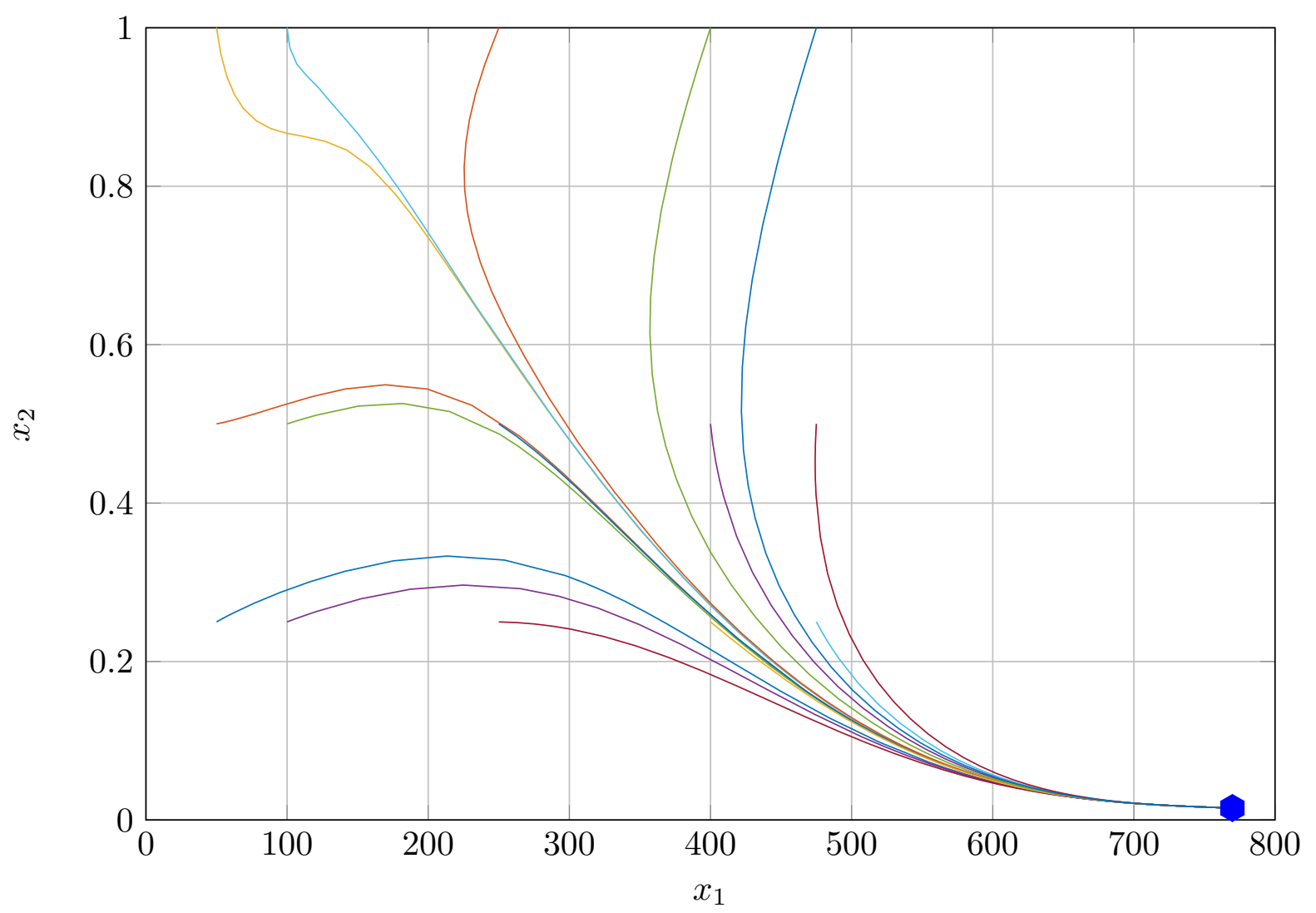

Figure 3: Example of the phase portrait of system (1), when its corresponding polynomial (6) has two complex roots.

The condition of Theorem 1 allows us to check the admissibility of a given parameters vector. In addition to the satisfaction of this condition, one can check the positivity of the real equilibrium points after solving (5).

Definition 3 (Admissibility of $p$ ) We say that a vector of parameters $p$ is admissible if the condition of Theorem 1 is satisfied and the real distinct roots of (6) are positive.

Definition 3 will be used in the sequel in the algorithm that we suggest to estimate the robust region of attraction of system (1). 


\subsection{Equilibrium points distribution}

Let's consider that the vector of model parameters $p$ is unknown and belong to the following interval:

$$
\left[0.9 p_{\text {nom }}, 1.1 p_{\text {nom }}\right]
$$

where $p_{\text {nom }}$ stand for the vector containing nominal parameters in Table 1. We can draw the distribution of the equilibrium points of model (1), using Monte-Carlo tests corresponding to random selections of the model parameters in the given interval.

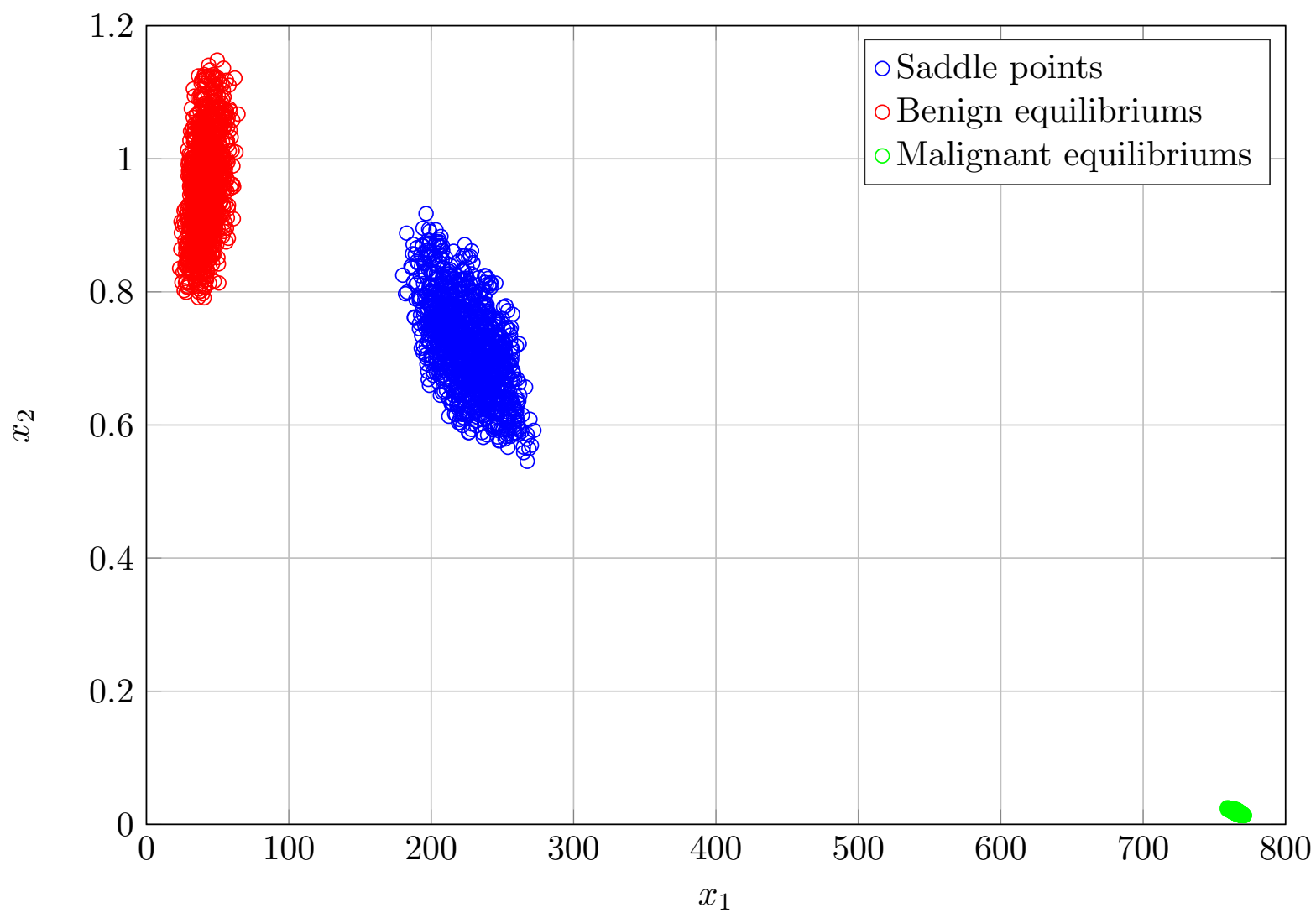

Figure 4: Distribution of equilibrium points under uncertainties, in red the benign equilibriums, in blue the saddle points and in green the malignant equilibriums.

Figure 4 shows the distribution of the equilibriums of system (1) for 1600 uniformally distributed samples of model parameters in the given interval. This figure shows that the malignant equilibrium points are considerably less dispersed than the benign ones and the saddle points. For this choice of uncertainties interval, all the selected parameters vectors were admissible.

The distribution of the benign equilibrium points presented in Figure 4 has been used in [20], in order to characterize a certified set where the state trajectories converge to their respective benign equilibriums in spite of all possible parametric uncertainties meeting (12). 


\section{RoA estimation with bang-bang control}

The cancer dynamical system (1) can be written as:

$$
\dot{x}=F(x, u, p), \quad x(0)=x_{0} .
$$

We denote by $\Phi_{u}\left(T, x_{0}, p\right)$ the solution of this system evaluated at time $T \geq 0$ for a given initial state $x_{0}$ using a control strategy $u(\cdot)$. Let's denote by $\Omega_{u}^{p}$ the controlled domain of attraction of system (1) with a bang-bang control strategy, for a given vector of parameters $p$. We consider the following state and input constraints sets:

$$
\begin{gathered}
\mathbb{X}=\left\{x \in \mathbb{R}_{+}^{2} \mid x_{2} \geq c\right\} \\
\mathbb{U}=\left\{u \in \mathbb{R}_{+}^{2} \mid u_{1}, u_{2} \in\{0,1\}\right\}
\end{gathered}
$$

The control input constraint set $\mathbb{U}$ in (15) allows to consider bang-bang control strategies.

Definition 4 The RoA $\Omega_{u}^{p}$ of the controlled system (1) is defined as follows:

$$
\Omega_{u}^{p}=\left\{x_{0} \in \mathbb{R}_{+}^{2} \mid \exists u(\cdot) \text { s.t. } \Phi_{u}\left(T, x_{0}, p\right) \in \Omega_{0}^{p}, x \in \mathbb{X}, u \in \mathbb{U}\right\} \text {. }
$$

where $\Omega_{0}^{p}$ is the previously defined region of attraction of the benign equilibrium $x_{b}^{p}$ of system (1), without drugs, corresponding to the admissible parameters vector $p$. We denote by $\mathbb{X} \subset \mathbb{R}^{n}$ and $\mathbb{U} \subset \mathbb{R}^{m}$ the sets of admissible values corresponding the state $x$ and the control $u$, respectively.

Practically, (16) means that we set a therapy time $T$, then we characterize the set of initial conditions $\Omega_{u}^{p}$ such that for each initial state $x_{0}$ (information about the patient health) belonging to $\Omega_{u}^{p}$, there exists at least one control law $u(\cdot)$, which allows to drive the states trajectories to the safe region $\Omega_{0}^{p}$ without violating the constraints on states and control inputs.

Problem 1 (Estimation of the nominal controlled RoA) Given the nominal parameters vector $p_{n o m}$ and considering bang-bang control strategies, characterize the region of attraction of the controlled system (1).

This region is denoted $\Omega_{u}^{p_{n o m}}$ and provides the set of state initial conditions for which there exists a bang-bang control strategy denoted $u(\cdot)$, such that the states at the end of the treatment period (at time $T$ ) belong to the region of attraction of the benign equilibrium $\Omega_{0}^{p_{n o m}}$ (the safe region for the nominal parameters vector $p_{\text {nom }}$ without control inputs). Additionally, the state trajectories as well as the control inputs have to satisfy the constraints defined by the sets $\mathbb{X}$ and $\mathbb{U}$. 
In this section, we present a methodology to estimate the region of attraction of system (1). Firstly, we characterize the domain of attraction for a given admissible parameters vector $p$. Then, in the next section, we provide a heuristic estimate of the robust region of attraction for model (1).

\subsection{Characterizing the RoA for the nominal controlled system}

Let's consider the vector of nominal parameters $p_{\text {nom }}$ and bang-bang control strategies. Since we have only two control inputs $u_{1}$ and $u_{2}$ corresponding to chemotherapy and immunotherapy injections respectively, there are only four possible instantaneous injection strategies. We inject only chemotherapy, only immunotherapy, both of them or neither chemotherapy nor immunotherapy.

Let's denote these injection strategies as follows:

$\mathcal{S}_{0,0}$ No drug injection $u=(0,0)$.

$\mathcal{S}_{1,0}$ Injection of chemotherapy $u=(1,0)$.

$\mathcal{S}_{0,1}$ Injection of immunotherapy $u=(0,1)$.

$\mathcal{S}_{1,1}$ Injection of both chemotherapy and immunotherapy $u=(1,1)$.

By drawing the phase portrait of the injection strategies previously listed, we can have an information on all possible bang-bang strategies allowing to drive the states to the safe region, without constraints violation. This can help us to derive an estimate the set $\Omega_{u}^{p_{n o m}}$ previously defined. This choice of strategies makes the constraints in (15) directly satisfied. The satisfaction of state constraints specified by (14) can be checked by drawing it in the phase portrait as well.

Figure 5 shows the phase portrait of system (1) using the drug injection schedules listed above. In this figure, we can notice that all the black trajectories corresponding to a continuous injection of chemotherapy violate the minimal constraint on immune cells density. We can notice also that the continuous injection of immunotherapy (represented by blue trajectories) allows to enlarge the domain of attraction of the benign equilibrium. Moreover, all the magenta trajectories, corresponding to a continuous injection of both chemoand immunotherapy, converge to the safe region, which further enlarges the domain of attraction of the benign equilibrium. However, we can notice that for bigger initial cancer volumes, the magenta trajectories violate the minimal constraint on immune cells density.

Figure 5 shows all the possibilities of switching between the different strategies in order to drive the states to the safe region. An interesting option is to choose the strategy allowing to reduce the quantity of injected drugs or to minimize the hospitalization time. We do not further investigate this idea here, since we are interested in estimating the domain of attraction of system (1). Therefore, the only relevant information is the existence of at least one control strategy allowing to drive the states to the region of attraction of the benign equilibrium. 


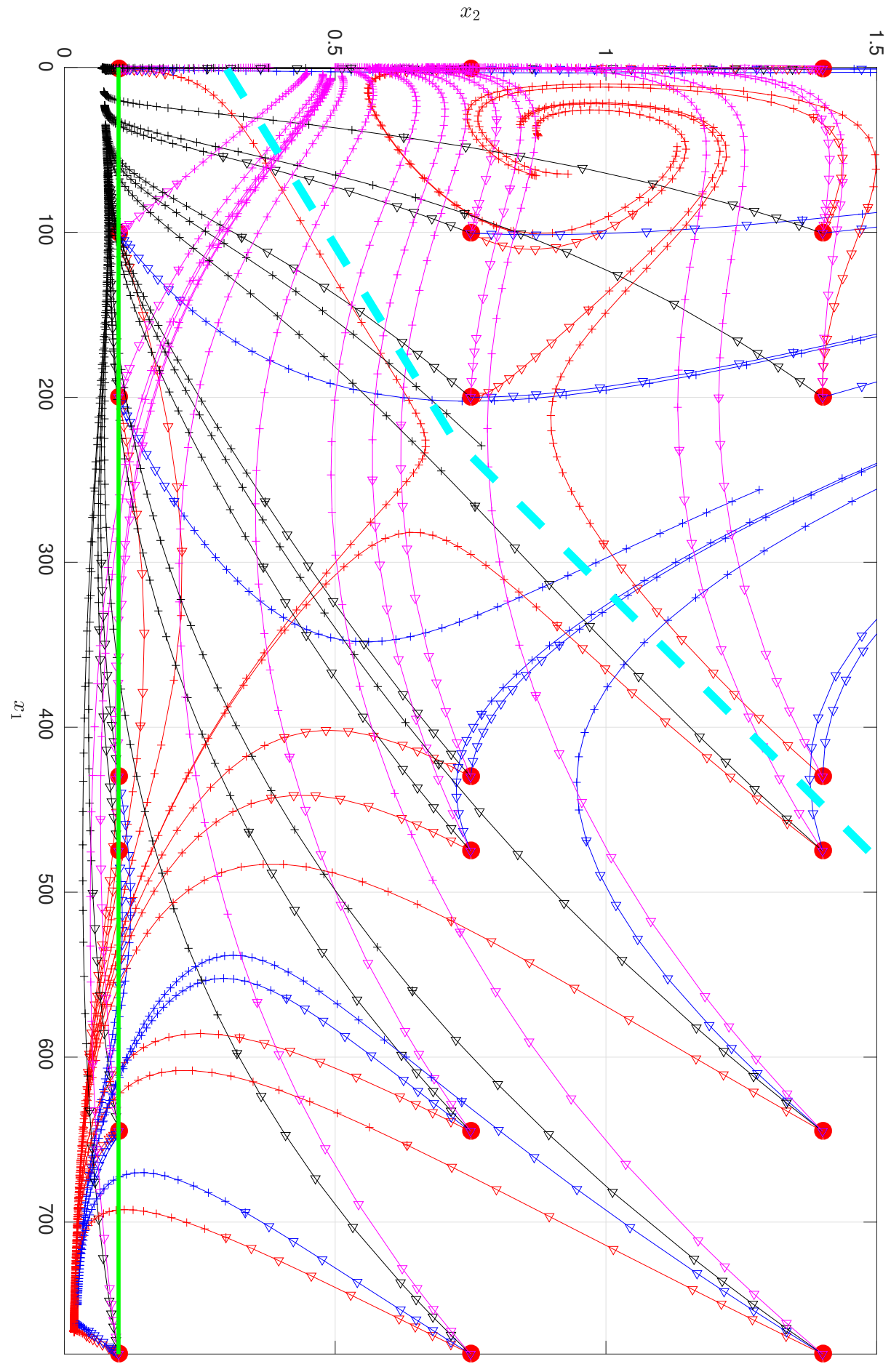

Figure 5: Phase portrait of system (1) with different drug injection strategies, the red trajectories correspond to $\mathcal{S}_{0,0}$, the black ones to $\mathcal{S}_{1,0}$, the blue ones to $\mathcal{S}_{0,1}$ and the magenta ones to $\mathcal{S}_{1,1}$, in green the minimal constraint on immune cells density, in dashed cyan the estimated nominal uncontrolled region of attraction of the benign equilibrium. The triangle sign denotes the beginning of a trajectory, whereas the sign + denotes its ending. 
Since the strategy of injecting both therapies provides the biggest domain of attraction, we focus on the magenta trajectory that is tangential to the minimal constraint $x_{2} \geq c$. This trajectory is depicted by (1) in Figure 6 , we can notice also that in this region of the state space, the blue trajectories (with immunotherapy only) evolve above the constraint line, before converging to the malignant equilibrium. We are interested in characterizing the blue trajectory that is tangential to the magenta one (depicted by (2) in Figure 6) in order to further enlarge the domain of attraction of the controlled system (1). Note that for this specific initial state (represented in green in Figure (6)), the strategy to consider is to use immunotherapy till the state reaches the yellow point and then to use both chemotherapy and immunotherapy in order to satisfy the specified constraint.

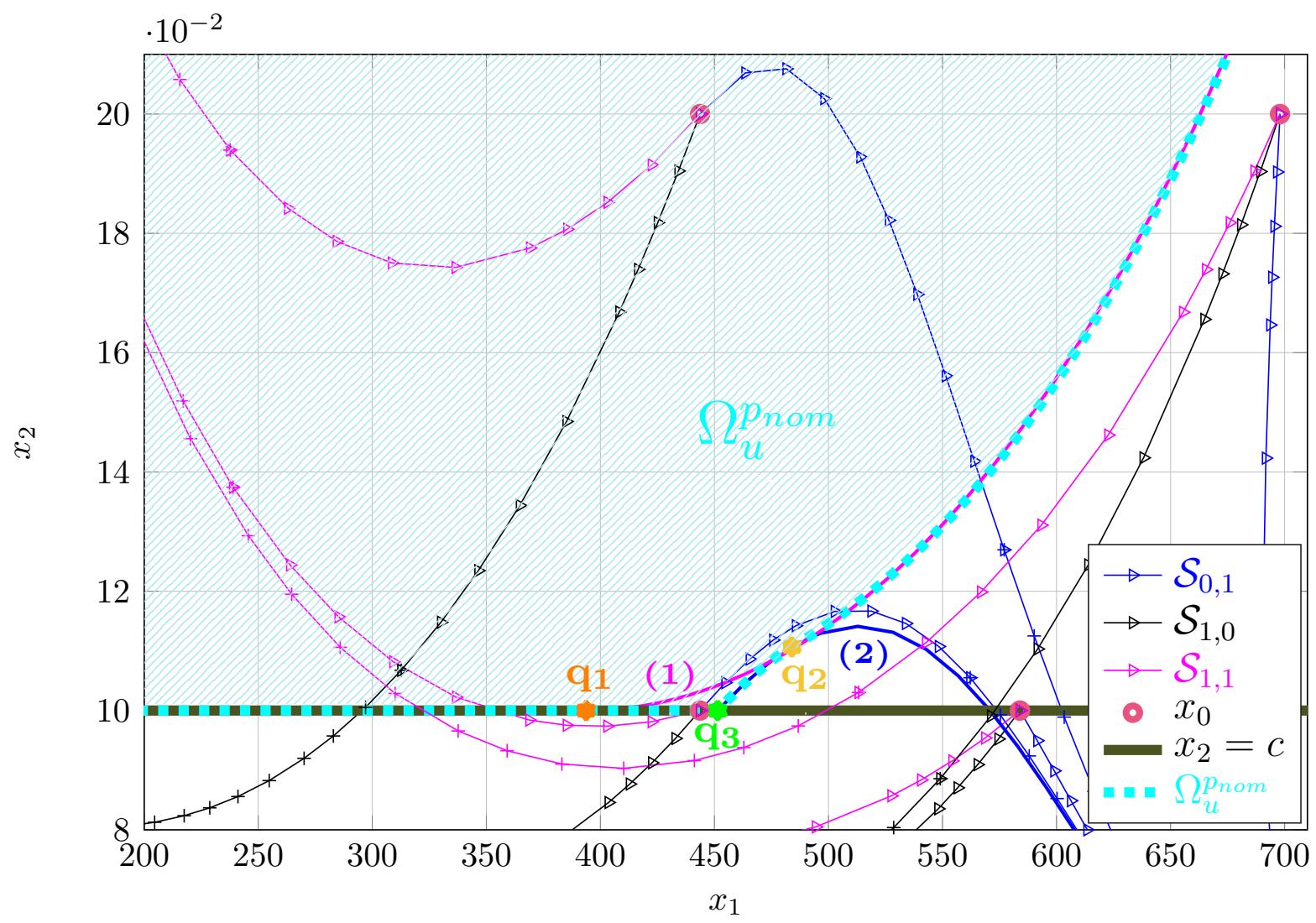

Figure 6: The three points $q_{1}, q_{2}$ and $q_{3}$ characterizing the RoA of model (1), and the resulting RoA that is an estimate of $\Omega_{u}^{p_{n o m}}$ using nominal parameters is shown in cyan dashed line. The triangle sign denotes the beginning of a trajectory, whereas the sign + denotes its ending.

Let's denote the points characterizing the domain of attraction as follows:

$\mathrm{q}_{1}=\left(\mathrm{q}_{11}, \mathrm{q}_{12}\right)$ The point where the magenta trajectory is tangential to the minimal constraint on immune cells density $x_{2} \geq c$.

$\mathrm{q}_{2}=\left(\mathrm{q}_{21}, \mathrm{q}_{22}\right)$ The point where the blue trajectory is tangential to the magenta one. 
$\mathrm{q}_{3}=\left(\mathrm{q}_{31}, \mathrm{q}_{32}\right)$ The point where the blue trajectory intersects with the constraint line.

In the sequel, we provide a generic methodology to derive the three points characterizing the domain of attraction of the controlled system (1).

\section{Computing $q_{1}$}

System (1) can be written as :

$$
\begin{aligned}
& \dot{x}_{1}=F_{1}(x, u, p), \\
& \dot{x}_{2}=F_{2}(x, u, p) .
\end{aligned}
$$

In order to find $q_{1}$ we have to solve $F_{2}=0$ for $x_{2}=c$ and $u=(1,1)$, it implies solving the following equation:

$$
-c \mu_{I} \beta_{Y} x_{1}^{2}+c \mu_{I} x_{1}+\alpha_{Y}-c \delta_{Y}+c \kappa_{Y}-c \eta_{Y}=0 .
$$

Let $q_{11}$ be the positive solution of (18), $q_{1}$ is defined as follows:

$$
q_{1}=\left(q_{11}, c\right)
$$

\section{Computing $q_{2}$}

Let's denote the time inverse trajectory of (13) for $u=(1,1)$, having as final point $q_{1}$, as $x_{2}=g\left(x_{1}\right)$ and $g_{p}$ as the polynomial approximation of $g$ up to some degree. In order to find $q_{2}$, we have to solve the following equations:

$$
\begin{aligned}
& F(x,(0,1), p) \times F(x,(1,1), p)=0, \\
& x_{2}=g_{p}\left(x_{1}\right) .
\end{aligned}
$$

Solving (20) implies solving the following equations system:

$$
\left\{\begin{array}{l}
-\mu_{I} \beta_{Y} \kappa_{X} x_{1}^{3} x_{2}+\left(\frac{\mu_{c}}{x_{\infty}}+\mu_{I} \kappa_{X}\right) x_{1}^{2} x_{2}+\gamma_{X} \eta_{Y} x_{1} x_{2}^{2}+\left(\kappa_{X} \kappa_{Y}-\delta_{Y} \kappa_{X}-\mu_{c} \eta_{Y}\right) x_{1} x_{2} \\
\quad+\kappa_{X} \alpha_{Y} x_{1}=0 \\
x_{2}=g_{p}\left(x_{1}\right)
\end{array}\right.
$$

Finally, solving (21) provides an approximation of $q_{2}$.

\section{Computing $q_{3}$}


Let $x_{2}=h\left(x_{1}\right)$ be the time inverse trajectory of $(13)$ for $u=(0,1)$, having as final point $q_{2}$. We denote by $x_{2}=h_{p}\left(x_{1}\right)$ the polynomial approximation of this trajectory. Therefore, in order to find $q_{3}$, we need to solve the following equation:

$$
h_{p}\left(x_{1}\right)-c=0 .
$$

Solving (22) provides an approximation of $q_{31}$ and $q_{3}$ is defined as follows:

$$
q_{3}=\left(q_{31}, c\right)
$$

Note that the solutions of the equations allowing to derive the points $q_{1}, q_{2}$ and $q_{3}$ depend on the parameters vector $p$. However, one can validate the RoA structure afterwards, by checking the following conditions:

$$
\begin{cases}q_{11}>0, & q_{31}>q_{11} \\ q_{21}>q_{31}, & q_{22}>c\end{cases}
$$

Remark 1 The methodology allowing to derive the points $q_{1}, q_{2}$ and $q_{3}$ has been tested over 2000 scenarios, corresponding to uncertain parameters vectors, as defined in (12). We also checked, using the conditions in (24), that all these scenarios have the same characterization shown in Figure 6.

\subsection{Algorithm for the estimation of domains of attraction}

After deriving the characteristic points as explained in the previous section, the region of attraction of the controlled system (1) is characterized by the following trajectory (see Figure 6):

$$
x_{2}=\mathcal{D}^{p}\left(x_{1}\right)= \begin{cases}c & \text { if } x_{1}<q_{31} \\ h_{p}\left(x_{1}\right) & \text { if } q_{31} \leq x_{1} \leq q_{21} \\ g_{p}\left(x_{1}\right) & \text { if } x_{1}>q_{21}\end{cases}
$$

The region of attraction $\Omega_{u}^{p}$ is defined as follows:

$$
\Omega_{u}^{p}=\left\{x \in \mathbb{R}^{2} \mid x_{2} \geq \mathcal{D}^{p}\left(x_{1}\right)\right\}
$$

Algorithm 1 summarizes the methodology previously explained, allowing to derive the domain of attraction of the controlled system (1) for a given vector of parameters $p$ and considering bang-bang control strategies.

Figure 6 shows the estimated domain of attraction of system (1) denoted $\Omega_{u}^{p_{n o m}}$, for nominal parameters $p_{n o m}$, that we obtained using Algorithm 1. It also shows the trajectories corresponding to $\mathcal{S}_{1,0}, \mathcal{S}_{0,1}$ and $\mathcal{S}_{1,1}$ for different initial states, highlighting the fact that the state trajectories starting out of the estimated region of attraction, either converge to the malignant equilibrium or violate the specified constraint. 


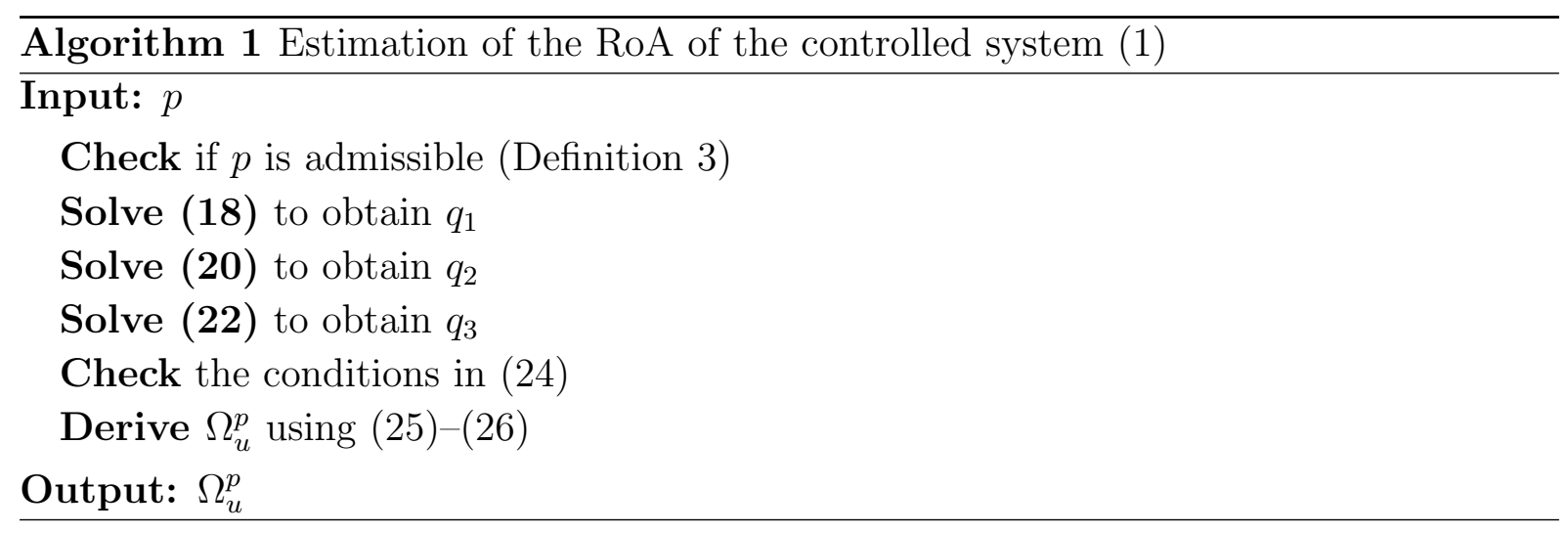

\subsection{RoA sensitivity analysis}

We showed in the previous section that the nominal domain of attraction of system (1) can be characterized by the points $q_{1}, q_{2}$ and $q_{3}$ (see Figure 6). In this section, we are interested in investigating the sensitivity of the RoA estimation with uncertainties on the model parameters. Therefore, we change the parameters values with some percentages, in order to see the effect of this change on the estimation of the ROA.

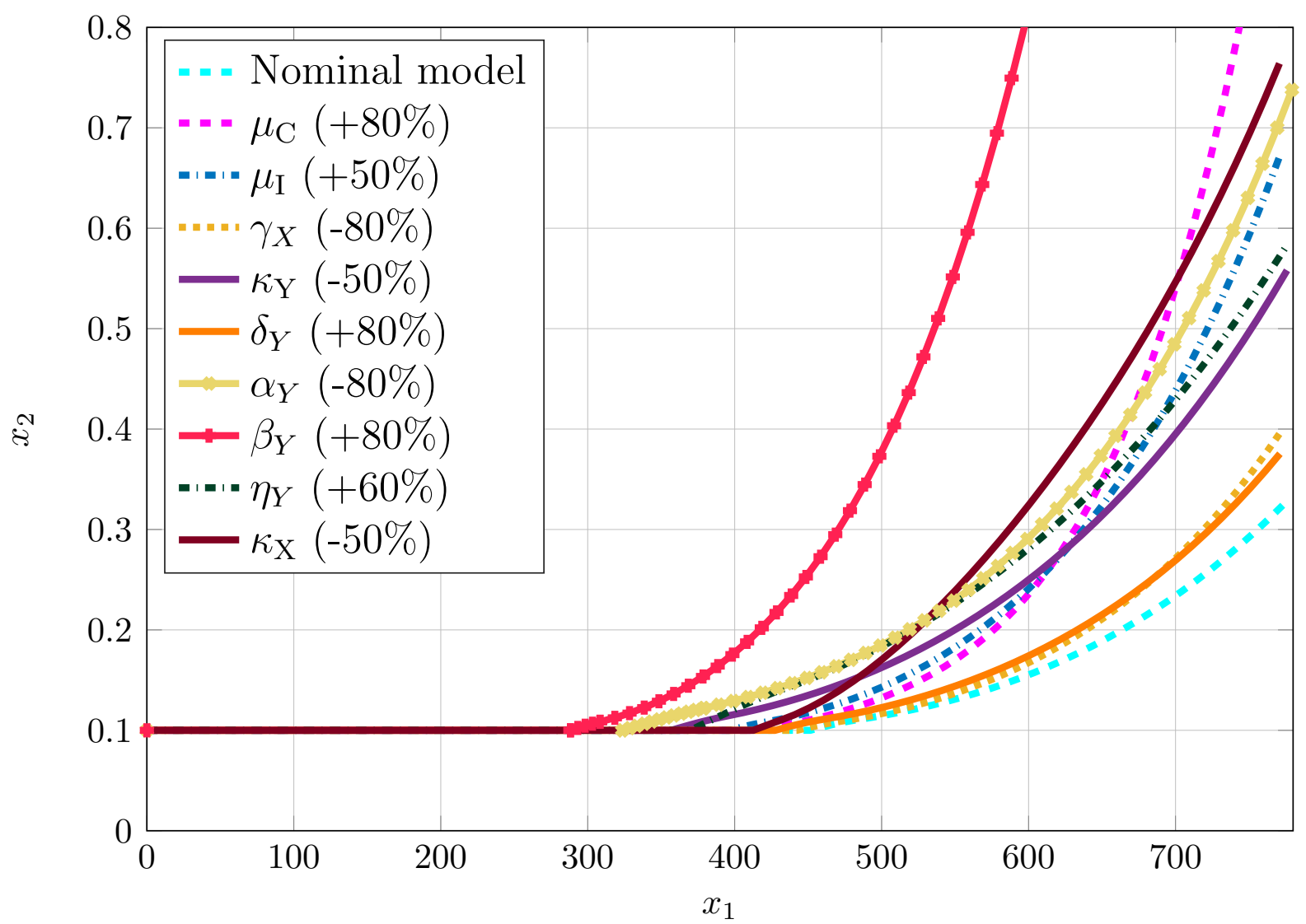

Figure 7: The sensitivity of RoA estimation with respect to the model parameters.

Figure 7 shows the nominal controlled RoA denoted $\Omega_{u}^{p_{n o m}}$ in cyan dashed line. This figure shows also the RoA of system (1) for different changes in the model parameters. 
We notice that changing the parameters $\delta_{Y}$ and $\gamma_{X}$ changes slightly the RoA estimation, whereas by changing the parameter $\beta_{Y}$ the RoA volume decreases drastically. The other parameters show more or less the same sensitivity.

Remark 2 The RoAs shown in Figure 7 are derived for deterministic parameters vectors, in the sense that we change one parameter value and derive the RoA for a fixed parameters vector.

Note that the parameters changing signs (either + or - ) have been chosen such that the RoA volume is reduced. Furthermore, the percentage of change has been chosen such that the parameters vector $p$ remains admissible.

\section{Heuristic estimate of the robust RoA}

The characterization of the domain of attraction of a given system as explained in Problem 1 is interesting since it provides the set of initial conditions that can be driven to the safe region. However the common assumption made for such deterministic approaches is that the system parameters are perfectly known [6], which is not realistic for practical problems. As previously mentioned, system parameters are generally affected by uncertainties that can be described by probability distributions or belong to given intervals.

After characterizing the region of attraction of system (1) for a given parameters vector, it is interesting to find the domain of attraction when the model parameters are uncertain. This set is called the robust region of attraction and represents the set of initial conditions that can be driven to the safe region in spite of all possible uncertainties. The robust region of attraction is defined as the intersection of all the regions of attraction governed by (1) for all possible realizations of $p[29]$.

Definition 5 The robust region of attraction of system (13), for a given set of parameters $\mathbb{P}$, denoted $\Omega_{u}^{\mathbb{P}}$ is defined as follows:

$$
\Omega_{u}^{\mathbb{P}}=\bigcap_{p \in \mathbb{P}} \Omega_{u}^{p}
$$

Remark 3 Note that this definition of the robust RoA means that there exists a control $u$ for each initial state $x_{0}$ and parameters vector $p$. This can be seen as an outer approximation of the real robust RoA, which is indeed bigger.

Problem 2 (Estimation of the robust controlled RoA) Given an uncertain parameters vector $p$ belonging to a set $\mathbb{P}$, we are interested in estimating the robust region of attraction of system (1), such that the state trajectories corresponding to the initial states in this set, belong to the safe region after some time and 
do not violate the specified constraints, in spite of all possible parametric uncertainties.

It is commonly known that finding the exact robust region of attraction for a nonlinear system is a challenging task. Therefore, we aim here at providing a tighter estimate of the robust region of attraction $\Omega_{u}^{\mathbb{P}}$ that we denote $\Omega_{R}$.

Let's denote by $\left\{p^{(j)}\right\}_{j=1}^{N}$ a collection of samples of the parameters vector $p$ corresponding to model (1), uniformly drawn in the following interval:

$$
\left[0.9 p_{\text {nom }}, 1.1 p_{\text {nom }}\right] \text {. }
$$

In the previous section, a characterization of the RoA of system (1) for nominal parameters $p_{\text {nom }}$ denoted by the set $\Omega_{u}^{p_{n o m}}$ had been provided. This procedure can be applied in order to derive the RoA of system (1) for each parameters sample $p^{(j)}$, by checking the validity of the characterization through the conditions (24).

We denote by $\Omega_{u}^{p^{(j)}}$ the RoA of the controlled system (1) considering the parameters vector $p^{(j)}$ and bang-bang control strategies.

In order to characterize the robust region of attraction, we perform $N$ Monte-Carlo tests assuming that the samples $\left\{p^{(j)}\right\}_{j=1}^{N}$ are uniformly distributed in the interval (28). The intersection of all the sets $\Omega_{u}^{p^{(j)}}$, estimated for each sample $p^{(j)}$, is defined by the maximum of all the corresponding functions $\mathcal{D}^{p^{(j)}}$ (defined in (25)). Thus, the estimated robust region of attraction is the following:

$$
\Omega_{R}=\left\{x \in \mathbb{R}^{2} \mid x_{2} \geq \mathcal{D}_{R}\left(x_{1}\right)\right\},
$$

where $\mathcal{D}_{R}\left(x_{1}\right)$ is defined as follows:

$$
\mathcal{D}_{R}\left(x_{1}\right)=\max \left(\mathcal{D}^{p^{(1)}}\left(x_{1}\right), \cdots, \mathcal{D}^{p^{(N)}}\left(x_{1}\right)\right)
$$

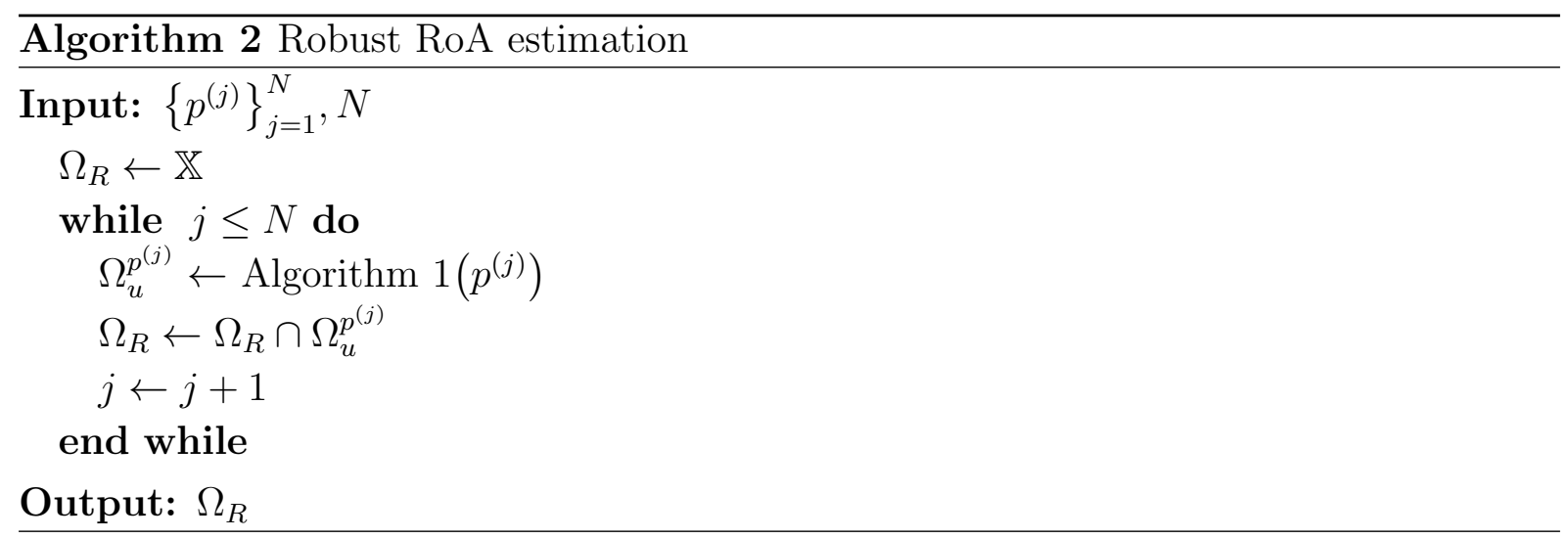

Algorithm 2 allows to derive a heuristic estimate of the robust region of attraction of system (1), for $N$ samples of parameters vectors, by intersecting their corresponding 
controlled regions of attraction.

Figure 8 shows the regions of attraction derived for $N$ samples $p^{(j)}$, the estimated robust region of attraction for different number of samples $N$, using Algorithm 2. We can notice that the estimation of $\Omega_{R}$ is enhanced and the robust RoA volume is reduced as the number of samples $N$ grows. We stopped running Algorithm 2 at $N=2000$ since for bigger values of $N$ the estimations of the robust RoA was almost the same as for $N=2000$. Note also that the estimated robust RoA is considerably smaller than the nominal one, even with the uncertainties rate that is only $\pm 10 \%$.

\section{Conclusion}

We presented in this paper an extensive parametric analysis for a cancer dynamical system. This allowed us to provide necessary and sufficient conditions for the admissibility of the model parameters vectors. Furthermore, we investigated the effects of parametric uncertainties on the system equilibrium points, as well as on the estimation of regions of attraction.

Therefore, we used a readily applicable methodology, allowing to characterize the domain of attraction of a nonlinear system describing cancer dynamics, with bang-bang control strategies. Then, we used this approach to derive an estimation of the robust region of attraction.

It is important to point out the fact that regions of attraction might be highly sensitive to parametric uncertainties, and can be considerably reduced when considering even small uncertainties on the model parameters. This is critical in the context of cancer treatment, since such sets provide an information on the patients that can be healed, using appropriate treatments. 


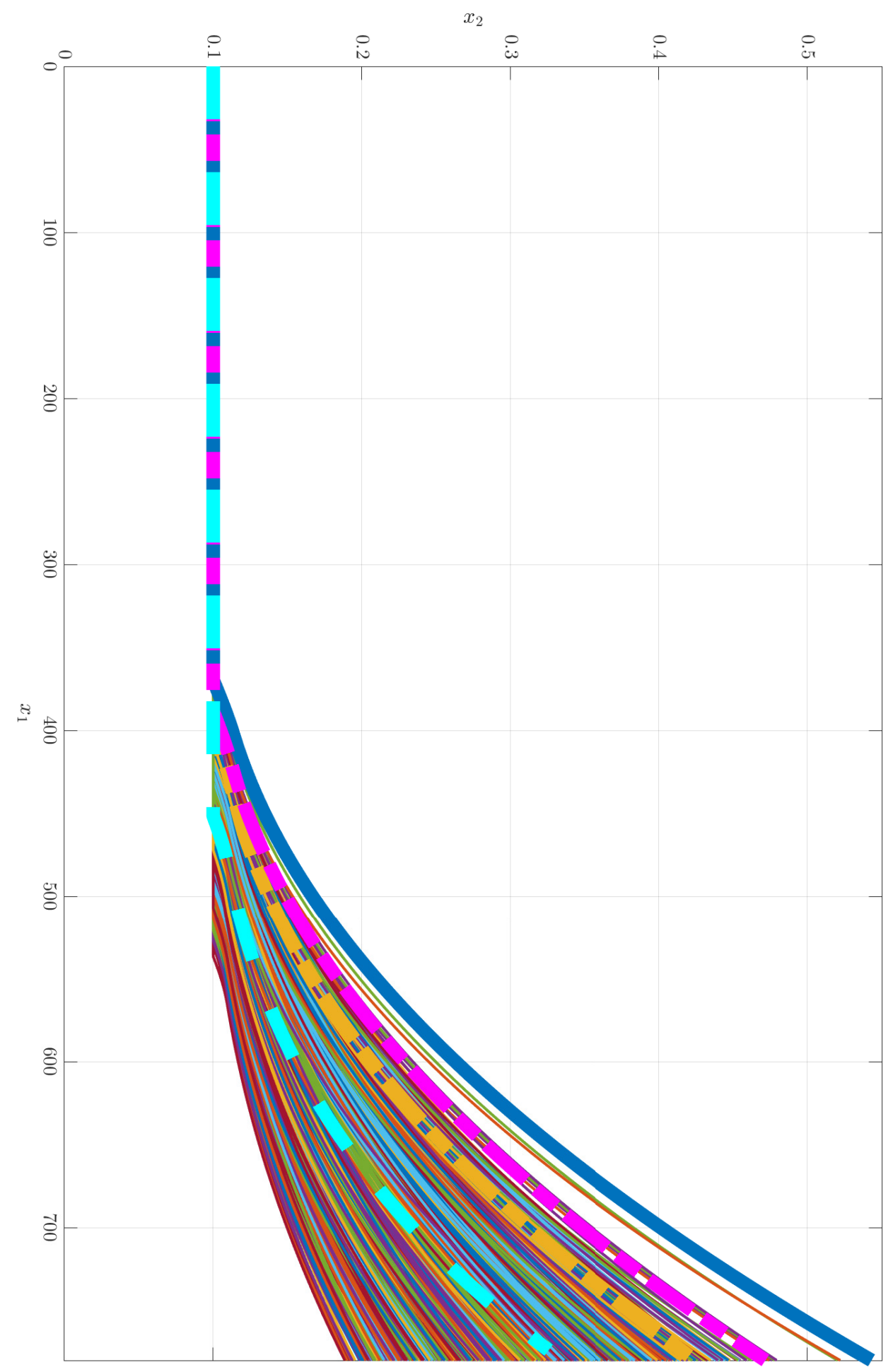

Figure 8: Monte-Carlo tests for the RoA estimation under $\pm 10 \%$ of parametric uncertainties, the blue bold trajectory defines the estimated robust region of attraction of system (1) denoted $\Omega_{R}$, for $N=2000$, the pink trajectory defines the estimated robust RoA for $N=1000$ and the orange one for $N=200$, the dashed cyan trajectory is the estimated nominal domain of attraction $\Omega_{u}^{p_{n o m}}$. 


\section{A Proof of Theorem 1}

Let's consider the following polynomial:

$a\left(x_{2}\right)=x_{2}^{3}+\mu_{C}\left(\frac{\mu_{I} x_{\infty} \gamma_{X}-2 \mu_{I} \beta_{Y} x_{\infty}^{2} \gamma_{X}}{\mu_{I} \beta_{X} x_{\infty}^{2} \gamma_{X}^{2}}\right) x_{2}^{2}+\mu_{C}^{2}\left(\frac{\delta_{Y}+\mu_{I} \beta_{Y} x_{\infty}^{2}-\mu_{I} x_{\infty}}{\mu_{I} \beta_{Y} x_{\infty}^{2} \gamma_{X}^{2}}\right) x_{2}-\frac{\mu_{C}^{2} \alpha_{Y}}{\mu_{I} \beta_{Y} x_{\infty}^{2} \gamma_{X}^{2}}$

Let $a_{2}, a_{1}$ and $a_{0}$ be the coefficients of the monic polynomial (31) such that:

$$
\left\{\begin{array}{l}
a_{2}=\mu_{C}\left(\frac{\mu_{I} x_{\infty} \gamma_{X}-2 \mu_{I} \beta_{Y} x_{\infty}^{2} \gamma_{X}}{\mu_{I} \beta_{X} x_{\infty}^{2} \gamma_{X}^{2}}\right) \\
a_{1}=\mu_{C}^{2}\left(\frac{\delta_{Y}+\mu_{I} \beta_{Y} x_{\infty}^{2}-\mu_{I} x_{\infty}}{\mu_{I} \beta_{Y} x_{\infty}^{2} \gamma_{X}^{2}}\right) \\
a_{0}=-\frac{\mu_{C}^{2} \alpha_{Y}}{\mu_{I} \beta_{Y} x_{\infty}^{2} \gamma_{X}^{2}}
\end{array}\right.
$$

Let $a_{p}=\left(a_{2}, a_{1}, a_{0}\right)^{T}$ be the coefficients vector corresponding to the polynomial $a\left(x_{2}\right)$. We can define the Hermite form corresponding to (31) as follows:

$$
\mathcal{H}\left(a_{p}\right)=\left(\begin{array}{lll}
s_{0} & s_{1} & s_{2} \\
s_{1} & s_{2} & s_{3} \\
s_{2} & s_{3} & s_{4}
\end{array}\right)
$$

where $s_{0}, s_{1}, s_{2}, s_{3}$ and $s_{4}$ are defined as follows:

$$
\left\{\begin{array}{l}
s_{0}=3 \\
s_{1}=-a_{2} \\
s_{2}=a_{2}^{2}-2 a_{1} \\
s_{3}=-a_{2}^{3}+3 a_{1} a_{2}-3 a_{0} \\
s_{4}=a_{2}^{4}-4 a_{1} a_{2}^{2}+2 a_{1}^{2}+4 a_{0} a_{2}
\end{array}\right.
$$

According to Theorem 1.1 in [23], the Hermit matrix $\mathcal{H}\left(a_{p}\right)$ is positive definite if and only if the roots of $a\left(x_{2}\right)$ are real and distinct.

\section{References}

[1] Alamo, T., Cepeda, A., Fiacchini, M., and Camacho, E. F. Convex invariant sets for discrete-time lur'e systems. Automatica 45 (2009), 1066-1071.

[2] Alamo, T., Cepeda, A., And Limon, D. Improved computation of ellipsoidal invariant sets for saturated control systems. In Proceedings of the 44th IEEE Conference on Decision and Control, and the European Control Conference (Sevilla, Spain, December 2005).

[3] Blanchini, F. Ultimate boundedness control for discrete-time uncertain systems via set-induced Lyapunov functions. IEEE Transactions on Automatic Control 39 (1994), 428-433. 
[4] Blanchini, F. Set invariance in control. Automatica 35, 11 (1999), 1747 - 1767.

[5] Blanchini, F., And Miani, S. Set-theoretic methods in control. Springer, 2008.

[6] Chesi, G. Estimating the domain of attraction for uncertain polynomial systems. Automatica 40 (2004), 1981-1986.

[7] Doban, A. I., And Lazar, M. Domain of attraction computation for tumor dynamics. In 53rd IEEE Conference on Decision and Control (2014), pp. 6987-6992.

[8] D'Onofrio, A., Ledzewicz, U., And Schättler, H. On the Dynamics of TumorImmune System Interactions and Combined Chemo- and Immunotherapy. Springer Milan, 2012, pp. 249-266.

[9] Farjami, S., Kirk, V., And Osinga, H. M. Computing the stable manifold of a saddle slow manifold. SIAM Journal on Applied Dynamical Systems 17, 1 (2018), 350-379.

[10] Fiacchini, M. Convex difference inclusions for systems analysis and design. $\mathrm{PhD}$ thesis, Universidad de Sevilla, Spain, 2012.

[11] Fiacchini, M., Alamo, T., and Camacho, E. F. On the computation of convex ro- bust control invariant sets for nonlinear systems. Automatica 46, 8 (2010), 13341338.

[12] Fiacchini, M., Alamo, T., and Camacho, E. F. Invariant sets computation for convex difference inclusions systems. Systems \& Control Letters 61, 8 (2012), 819-826.

[13] Fiacchini, M., Tarbouriech, S., and Prieur, C. Polytopic control invariant sets for differential inclusion systems : a viability theory approach. In Proceedings of the 2011 American Control Conference ACC (2011), pp. 1218-1223.

[14] Francomano, E., Hilker, F. M., Paliaga, M., and Venturinoc, E. An efficient method to reconstruct invariant manifolds of saddle points. Dolomities Research Notes on Apptoximation, Padova University Press 10 (2017), 25-30.

[15] Henrion, D., And Korda, M. Convex computation of the region of attraction of polynomial control systems. IEEE Transactions on Automatic Control 59, 2 (2014), 297-312.

[16] Korda, M., Henrion, D., And Jones, C. N. Inner approximations of the region of attraction for polynomial dynamical systems. IFAC Proceedings Volumes (IFACPapersOnline) 9, 1 (2013), 534-539.

[17] Ledzewicz, U., ANd FAraJi, M. On optimal protocols for combinations of chemoand immunotherapy. In 51st IEEE Conference on Decision and Control (Hawaii, USA, December 2015), pp. 7492-7497. 
[18] Ledzewicz, U., And Schättler, H. On the Role of the Objective in the Optimization of Compartmental Models for Biomedical Therapies. Journal of Optimization Theory and Applications (2020).

[19] Merola, A., Cosentino, C., and Amato, F. An insight into tumor dormancy equilibrium via the analysis of its domain of attraction. Biomedical Signal Processing and Control 3, 3 (2008), $212-219$.

[20] Moussa, K., Fiacchini, M., And Alamir, M. Probabilistically Certified Region of Attraction of a Tumor Growth Model with Combined Chemo- and Immunotherapy. https://arxiv.org/pdf/2004.1407\%.pdf.

[21] Moussa, K., Fiacchini, M., And Alamir, M. Robust optimal control-based design of combined chemo- and immunotherapy delivery profiles. In The 8th IFAC Conference on Foundations of Systems Biology in Engineering (València, Spain, October 15-18 2019).

[22] Moussa, K., Fiacchini, M., and Alamir, M. Robust optimal scheduling of combined chemo- and immunotherapy: Considerations on chemotherapy detrimental effects. In The 2020 American Control Conference (Denver, USA, July 1-3 2020).

[23] Netzer, T., Plaumann, D., and Thom, A. Determinantal representations and the hermite matrix. Michigan Math. J. 62 (2013), 407-420.

[24] Riah, R. Théorie des ensembles pour le contrôle robuste des systèmes non linéaires: Application à la chimiothérapie et les thérapies anti-angiogéniques. $\mathrm{PhD}$ thesis, Communauté Université Grenoble Alpes, 2016.

[25] Riah, R., Fiacchini, M., And Alamir, M. Iterative method for estimating the robust domains of attraction of non-linear systems: Application to cancer chemotherapy model with parametric uncertainties. European Journal of Control 47 (2019), $64-73$.

[26] Sharifi, N., Ozgoli, S., And Ramezani, A. Multiple model predictive control for optimal drug administration of mixed immunotherapy and chemotherapy of tumours. Computer Methods and Programs in Biomedicine 144 (2017), 13-19.

[27] Sharifi, N., Zhou, Y., and Holmes, G., C. Y. Overcoming channel uncertainties in touchable molecular communication for direct drug targeting assisted immunochemotherapy. IEEE Transactions on NanoBioscience 19, 2 (2020), 249-258.

[28] Stepanova, N. Course of the immune reaction during the development of a malignant tumour. Biophysics (1980).

[29] Topcu, U., Packard, A. K., Seiler, P., and Balas, G. J. Robust Region-ofAttraction Estimation. IEEE Transaction on Automatic Control 55, 1 (2010). 
[30] Zarei, M., Javadi, K., And Kalhor, A. Perturbed tumor immunotherapy domain of attraction estimation via the arc-length function. In 2018 25th National and 3rd International Iranian Conference on Biomedical Engineering (ICBME) (2018), pp. 1-6.

[31] Zhou, B., Duan, G., And Lin, Z. Approximation and monotonicity of the maximal invariant ellipsoid for discrete-time systems by bounded controls. IEEE Transactions on Automatic Control 55, 2 (2010). 